\title{
Death of a young mother after sixth delivery due to coronary artery dissection
}

\author{
SenanayakeSMHMK
}

\begin{abstract}
Thirty six (36) years old female developed burning chest pain, was treated for gastritis by the general practitioner and was found dead on admission to the hospital 12 hours later. Postmortem examination showed hemorrhage around left coronary artery, left circumflex artery, anterior descending artery and branches, inner walls of coronary arteries compressed and lumens were collapsed. Lateral wall of the left ventricle showed pale area with hemorrhages. Cause of death was established as pulmonary oedema due to myocardial infarction following coronary artery dissection during puerperium. Most common causes for coronary artery dissection are coronary atherosclerosis and spontaneous peri-partum dissection. In peri-partum coronary artery dissections eosinophilic infiltration are seen in coronary adventitia without atherosclerosis. High estrogen levels or eosinophilic granulations contain lytic substances are thought to break down medial-adventitial layers leading to coronary artery dissection. Spontaneous coronary artery dissection occurs in relatively younger population with a predilection for women. The mean age of patients is 35 to 40 years. Left anterior descending coronary artery was the most common to involve. Some cases are diagnosed at the autopsy due to sudden deaths and some cases are presented as unstable angina and myocardial infarction. Spontaneous coronary artery dissection is a coronary abnormality needs attention in autopsies of any young person with history of chest pain or sudden death, especially women in the peri-partum period.
\end{abstract}

\section{Full paper}

\section{Introduction}

Maternal deaths are very important because it creates severe burden for the family, leads to health department inquiry about the clinical and antenatal managements, demand a post autopsy discussion by the relations with forensic medicine practitioner. During postmortem examinations of maternal deaths, rare causes of deaths are frequently found due to better antenatal care and obstetric managements. Even though coronary artery dissection is a rare cause of sudden death, it is an important cause of maternal deaths because most common causes for coronary artery dissection are coronary atherosclerosis and spontaneous peri-partum dissection. $^{[1]}$

Consultant Judicial Medical officer, Teaching Hospital Anuradhapura

Corresponding author: Senanayake SMHMK, email and phone number

DOI: http://doi.org/10.4038/mljsl.v4i2.7339

\section{Case report}

Thirty six years old female developed burning pain in chest and throat on 18th day after 6th normal delivery. She was treated for gastritis by the general practitioner and was reassured that investigations are not necessary at the moment. She was advised to come on next day for review. In the same day, mid night (after about 12 hours of onset of chest pain) she developed faintness and difficulty in breathing and was found dead on admission to the hospital.

Relations accepted that the woman had been given dates for permanent family planning method (ligation and resection of tubes) after 4th and 5th deliveries but could not attend due to illnesses of her children.

Postmortem examination showed followings. Pericardial cavity with $90 \mathrm{ml}$ of clear fluid. Hemorrhage along the surface of anterior descending artery and left circumflex artery (fig.1). In cut sections bleeding around left coronary artery (fig.2), anterior descending artery and main branch of it, left circumflex artery and main branch of it. First 3mm of 
coronary arteries compressed and lumen were blocked. Lateral wall of left ventricle showed pale area with hemorrhages compatible with acute myocardial infarction (fig.3). Bilateral massive pulmonary oedema was found. Histology showed blood in the walls of coronary arteries (fig, 4).Cause of death was

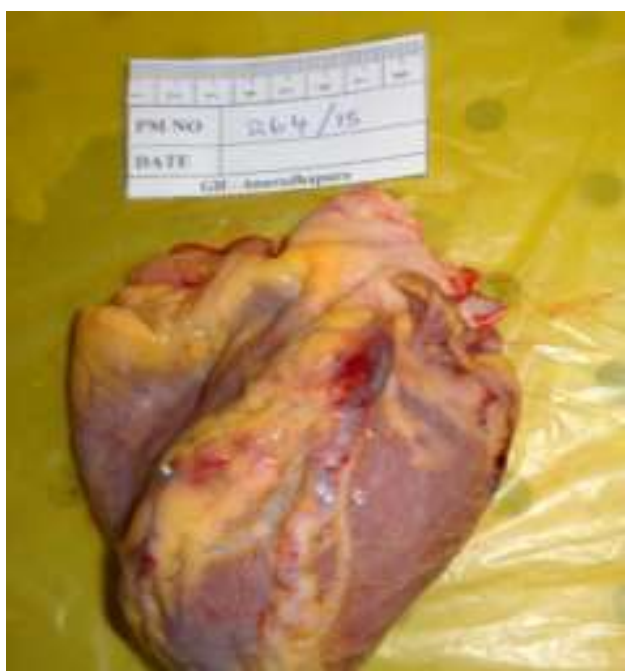

Figure1. Externally, hemorrhage along anterior descending artery

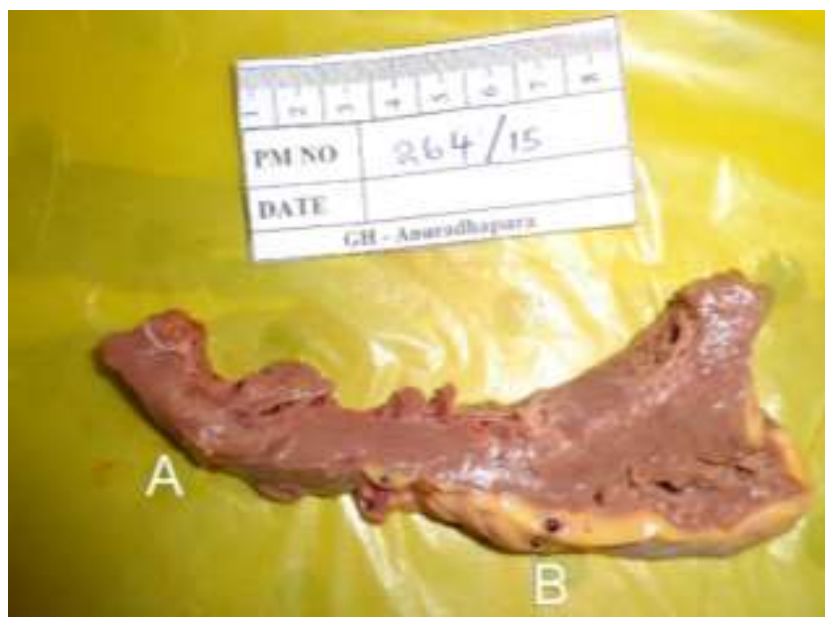

Figure3. Myocardial infarction of the left ventricle (A- Pale area, B- anterior descending artery with blood in the wall)

\section{Discussion}

Post autopsy discussion started with explaining the cause of death in general words. Relations raised two established as myocardial infarction due to spontaneous coronary artery dissection during the puerperium.

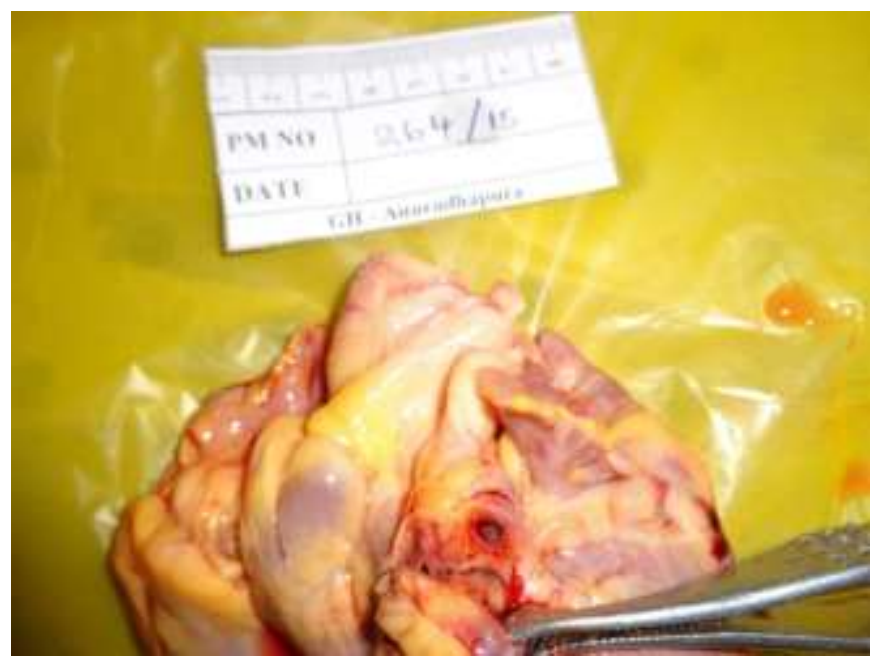

Figure 2. Cut section of anterior descending artery with collapsed lumen

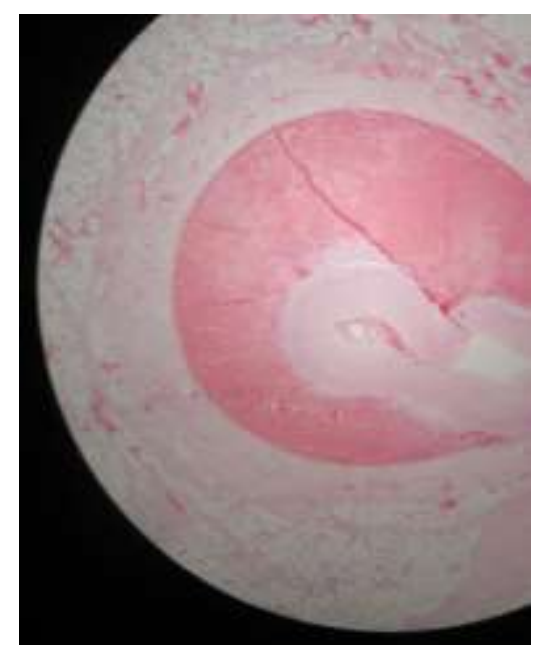

Figure 4. Microscopy of coronary artery dissection showing collapsed lumen and $\mathrm{RBC}$ in the wall 
questions. They were answered in a later date on appointment, after preparation and according to ethical guidelines. ${ }^{[2,3]}$

(1) "What is the reason for the illness"?

Coronary artery dissection as the cause of myocardial infarction is very rare occurrence among other causes such as thrombosis over coronary atheroma, embolism, spasm and bleeding in to atheromatous patch.

Most common causes for coronary artery dissection are coronary atherosclerosis and spontaneous dissection during peri-partum period. Peri-partum spontaneous coronary artery dissection shows eosinophilic infiltration in coronary adventitia without atherosclerosis. High estrogen levels or eosinophilic granulations contain lytic substances are thought to break down medial-adventitial layers leading to coronary artery dissection. ${ }^{[1]}$

Spontaneous dissection occurs in relatively younger population with a predilection for women. The mean age of patients is 35 to 40 years. Left anterior descending coronary artery was most common to involve. ${ }^{[4]}$ Some cases are diagnosed at the autopsy due to sudden deaths and some cases are presented as unstable angina and myocardial infarction. This case had presented with chest pain was managed as gastritis. Pale myocardium of infarcted area in the lateral wall of left ventricle indicates that she had lived several hours after the onset of myocardial infarction ${ }^{[5]}$ which was consistent with the history.

Coronary artery dissection may occur as a consequence of trauma, connective tissue disorder, cardiac surgery, coronary angiography or as an extension of aortic dissection.

In this case, because no cause was found and coronary arteries were free of atheroma, spontaneous peri partum coronary artery dissection was explained as the reason.

(2) "Whether investigations could have saved the life?"

If coronary artery dissection presents as unstable angina or myocardial infarction treatment options are available. ${ }^{[6]}$ General practitioner had a provisional diagnosis of gastritis and not planned for any urgent investigation. ECG test could have shown changes of cardiac pathology and at least patient could have been sent for inward management. The reason for omission of investigation is a clinical issue and need to be addressed by clinicians. ${ }^{[7,8]}$ Diagnosis and management of coronary artery dissection and saving the life depend on the severity, availability of cardiology unit and facilities. ${ }^{[9]}$ But if patient had followed the medical advice for family planning after fourth and fifth deliveries as advised, there would not be sixth pregnancy, puerperium and spontaneous dissection of coronary artery.

\section{Conclusion}

Spontaneous coronary artery dissection is a coronary abnormality needs attention in autopsies of any young person with history of chest pain or sudden deaths, especially women in the peri-partum period. Forensic medicine practitioners should participate in post autopsy discussions with sufficient preparation and according to ethical guidelines.

\section{References}

1. Robinowitz M, Virmani R, McAllister $\mathrm{H}$. Spontaneous coronary artery dissection and eosinophilic inflammation: A cause and effect relationship? American Journal of Medicine. 1982; 72:923-928.

2. Plueckhahn VD, Cordner SM. Ethics, Legal Medicine and Forensic Pathology. $2^{\text {nd }}$ edition, Victoria: Melbourne University Press;1991. pp-168169

3. Perera BPP. To the Editors: Should the Judicial Medical Officer (JMO) discuss the cause and manner of death with the family of the deceased? Ceylon Medical Journal. 2014; 59: 32

4. DeMaio Jr SM, Kinsella SH, Silverman ME. Clinical course and long-term prognosis of spontaneous coronary artery dissection. The American Journal of Cardiology. 1989;64(8):471-474 
5. Spitz WU. Asphyxia. In: Spitz WU editor. Spitz and fisher's Medicolegal Investigation of Death. 4th ed. Illinois; Charles C Thomas: 2004: p 1097

6.Tanis W, Stella PR, Pijlman AH, Kirkels JH, Peters RHJ, de Man FH. Spontaneous coronary artery dissections: current insights and therapy. Netherlands heart Journal. 2008;16 (10): 344-349

7. Varun G. Redressing Grievances and Complaints Regarding Basic Service Delivery. World Development.2013;January (41): 109-119
8.Ganapati M. Indian doctors are not accountable, says consumer report. British Medical Journal September 2000; 321: 588

9.Auer J, Punzengruber C, Berent $\mathrm{R}$, et al. Spontaneous coronary artery dissection involving the left main stem: Assessment by intravascular ultrasound. Heart. 2004; 90 (7):39. 